\title{
Unveiling Positioning Nystagmus in Patients of Horizontal Semicircular Canal Benign Paroxysmal Positional Vertigo by Diagnostic Head-Shaking in the Yaw Plane
}

\author{
Ajay Kumar Vats ${ }^{1}$ \\ ${ }^{1}$ Chaudhary Hospital and Medical Research Centre Private Limited, \\ Udaipur, Rajasthan, India
}

Ann Otol Neurotol:2019;2:85-88
Address for correspondence Ajay Kumar Vats, MBBS, MD (Medicine), DM (Neurology), Chaudhary Hospital and Medical Research Centre Private Limited, 472-473, Sector 4, Hiran Magri, Udaipur 313002, Rajasthan, India (e-mail: vatsneuro@gmail.com).

\begin{abstract}
Keywords

- benign paroxysmal positional vertigo

- Dix-Hallpike test

- Gufoni maneuver

- positional nystagmus

- supine roll test

Introduction The diagnosis of benign paroxysmal positional vertigo (BPPV) is largely dependent on elicitation of positioning nystagmus on the diagnostic positional tests, namely Dix-Hallpike and supine roll tests (DHT and SRT, respectively), in patients complaining of vertigo, which occurs when patient's head moves relative to the gravity. The pattern of elicited positioning nystagmus localizes as well as lateralizes the diseased canal, and the therapeutic positioning maneuver is accordingly undertaken.

Objective The diagnostic positional tests, at times fail to elicit positional nystagmus, leaving clinician in a state of dilemma, when examining a patient who is currently experiencing paroxysms of vertigo triggered by positional change. In two patients with history consistent with BPPV but with negative positional tests initially, head shaking for 10 seconds in the yaw axis was done, and Dix-Hallpike and supine roll tests were repeated. The aim of head shaking for 10 seconds was to unveil positional nystagmus, to precisely localize and lateralize the diseased semicircular canal.

Results and Discussion In the two cases of horizontal semicircular canal BPPV (HSC-BPPV) reported here, the DHT and/or SRT initially failed to elicit positional nystagmus but head shaking for 10 seconds in the left Dix-Hallpike position in case one and with the head anteflexed 30-degrees in the sitting position in the case two, unveiled horizontal positional nystagmus on ensuing SRT. The use of head-shaking in the yaw plane to unveil a horizontal positioning nystagmus in cases where a conventional positional test (DHT and SRT) has failed to elicit the PN, has not been reported in the literature hitherto.

Conclusion After precise localization and lateralization of the diseased canal, both patients successfully underwent successful treatment with Gufoni maneuver. A verifying SRT done at 1 hour and/or at 24 hours follow-up was negative. In patients, who are currently experiencing paroxysms of vertigo triggered by the change of position of head relative to the gravity; head-shaking for few seconds just prior to the positioning test, can unveil positional nystagmus not elucidated with the conventionally performed positional tests.
\end{abstract}

Copyright @2019 Indian Society of Otology
License terms

(요 (1) $\ominus \circledast$ 


\section{Introduction}

The diagnosis, localization, and lateralization of benign paroxysmal positional vertigo (BPPV) is largely dependent on the elicitation of positioning nystagmus on the diagnostic positional tests, namely Dix-Hallpike and supine roll tests (DHT and SRT respectively), in patients complaining of vertigo that occurs when patient's head moves in relation to gravity. The typical situations in which BPPV attacks occur are lying supine on bed, assuming lateral recumbent positions, extending neck, and bending forward. However, the diagnostic positional tests at times fail to show positioning nystagmus, even if meticulously sought. Such a situation leaves the clinician in a state of dilemma when examining a patient who is currently experiencing paroxysms of vertigo triggered by positional change. The elicitation of positional nystagmus (PN) was considered mandatory by Dix and Hallpike, who were first to develop objective criteria for BPPV diagnosis. ${ }^{1}$ The Bárány society has classified the cases of canalolithiasis of the posterior semicircular canal (PSC) without PN as "Possible benign paroxysmal positional vertigo" while all other variants of BPPV without elicitable PN, that are not attributable to posterior canalolithiasis are lumped under an ambiguous heading of "Probable benign paroxysmal positional vertigo, spontaneously resolved." ${ }^{2}$ However, all efforts should be made to unveil a PN before arriving to such a diagnosis, as ability to elicit it is not merely reassuring to the clinician in arriving at an accurate diagnosis but also precisely guides the treatment, which involves repositioning maneuvers, in which the head along with body of the patient, diagnosed to have BPPV, are sequentially oriented in such a manner that the otoconial debris is moved from its ostensible location within the involved semicircular canal under the gravitational force toward the utricle by observing the patterns of nystagmus elicited on the diagnostic positional tests. I report here two cases of horizontal semicircular canal BPPV (HSC-BPPV), in whom initially both DHT and/or SRT did not reveal positioning nystagmus but head shaking in the yaw plane elicited horizontal PN on the positional test ensuing within a minute after head shaking. Head shaking in yaw plane to increase the diagnostic yield of positional test in the HSC-BPPV has not been reported hitherto and this is the first case reporting of two such cases who failed to show PN on the conventional diagnostic positional tests. The diagnostic head-shaking for 10 seconds led to the diagnosis of this purely clinical vestibular disorder.

\section{Case 1 Description}

A 35-year-old male patient was seen on February 15, 2019, with 3 days history of intermittent true external vertigo lasting less than a minute on lying supine on the bed, assuming supine to sitting position as well as on assuming left lateral recumbent position. There was no history of staggering, diplopia, dysarthria, difficulty in swallowing, hiccups, drooping of upper eyelids, facial, or limb weakness. The neurological examination revealed normal cranial nerve examination, power was grade $5 / 5$ in all four limbs with normal deep tendon reflexes, and bilateral plantar reflexes were flexor. The examination of cerebellar system revealed no spontaneous or gaze evoked nystagmus and no appendicular or axial incoordination was observed. The oto-neurological examination revealed normal vertical and horizontal saccadic and smooth pursuit eye movements. The head impulse test was bilaterally normal. The Dix-Hallpike and supine roll tests done initially on both sides did not reveal any type of positioning nystagmus ( $\boldsymbol{- V i d e o s} \mathbf{1}$ and $\mathbf{2}$ ). As the patient was suspected to have left PSC involvement on the basis of history of vertigo on assuming left rather than both lateral recumbent positions as well on assuming sitting position from the supine and vice versa, the patient's head was taken to left Dix-Hallpike position and was briskly shaken side to side in yaw plane for 10 seconds but no positioning nystagmus could be elicited immediately on stopping the head shake ( - Video 3). As the suspicion of left PSC benign paroxysmal positional vertigo (PSC-BPPV) was very strong, the DixHallpike test to left was repeated again third time, within 1 minute of head shaking, which unexpectedly showed an apogeotropic horizontal positioning nystagmus lasting around 12 seconds ( - Video 4 ). As the horizontal positioning nystagmus occurs with the involvement of the HSC, it was decided to do a repeat supine roll test. The repeat supine roll test showed a geotropic PN on the left as well as to the right side (-Video 5). The visibly stronger geotropic PN on the left lateralized as well as localized the diseased canal to be the left HSC, with possible long posterior arm horizontal canalolithiasis. A diagnosis of left HSC-BPPV (geotropic variant) was established and patient was treated with two sequences of Gufoni maneuver at an interval of 1 hour. The Gufoni maneuver for the left geotropic HSC-BPPV was performed by making the patient sit on the edge of the couch with both lower limbs dangling down and briskly moving the patient from sitting to right (contralesional) lateral recumbent position and maintaining the latter position for 1 minute. Thereafter, patient's head was turned $\sim 45^{\circ}$ downward in the yaw plane and maintained for 2 minutes, after which he was taken to the upright sitting position (-Video $\mathbf{6}$ ). The supine roll test performed after 1 hour of treatment with second sequence of Gufoni maneuver did not show any nystagmus on either side ( -Video 7). The patient was telephonically contacted after 24 hours and he reported himself to be vertigo free.

\section{Case 2 Description}

A 48-year-old male patient was seen on May 28, 2019 with 1 day-history of intermittent true external vertigo lasting less than a minute, triggered by lying on bed, assuming supine to sitting as well as either of the lateral recumbent positions. There was no history of staggering, diplopia, dysarthria, difficulty in swallowing, hiccups, drooping of upper eyelids, facial or limb weakness. The neurological examination revealed normal cranial nerve examination, power was grade $5 / 5$ in all four limbs with normal deep tendon reflexes, and bilateral plantar reflexes were flexor. The examination of cerebellar system revealed no spontaneous or gaze evoked nystagmus and no appendicular or axial incoordination was 
observed. The oto-neurological examination revealed normal vertical and horizontal saccadic and smooth pursuit eye movements. The head impulse test was bilaterally normal. The supine roll tests done initially on both sides did not reveal any type of PN ( - Video 8). The patient was made to sit again with both lower limbs placed along the long axis of the examination couch. His head was anteflexed 30 degrees in the pitch plane and was briskly shaken in excursions of 30 degrees side-to-side in the yaw plane for around 10 seconds. Immediately, thereafter he was taken to supine neutral position on a 4-inch-thick pillow so that as his head landed on the pillow it got 30 degrees anteflexed. The head was briskly rotated to the left and maintained for $10 \mathrm{sec}-$ onds and as no PN was seen, it was then brought back to the neutral position. Then it was briskly rotated to his right and maintained for 15 seconds and as no PN was seen, it was then brought back to the neutral position. Maintaining the patient in supine position, the SRT was again repeated first to the left and then to the right and at this time a geotropic PN was elicited on both sides, which was visibly stronger on the right compared with the left, localizing the diseased canal as the right H-SCC ( -Video 9). The patient was treated with Gufoni maneuvers twice at an interval of 1 hour, for the geotropic variant of the right HSC-BPPV. The Gufoni maneuver for the right geotropic HSC-BPPV was performed by making the patient sit on the edge of the couch with both lower limbs dangling down and briskly moving the patient from sitting to left (contralesional) lateral recumbent position and maintaining the latter position for 1 minute. Thereafter, patient's head was rotated $\sim 45$ degrees downward in the yaw plane and maintained for 2 minutes, after which he was taken to the upright sitting position ( - Video 10). A supine roll test done at 1 and at 24 hours ( - Videos 11 and 12) after the second sequence of Gufoni maneuver did not show any PN indicating cure.

\section{Discussion}

A small amount of statoconia is believed to be present in the semicircular canals (SCC) of asymptomatic individuals, which are absorbed within few hours or days, without triggering any symptoms. A paroxysm of positional vertigo is triggered during head movement only when more otoconial debris from the utricular macula enters the SCC and increases the otoconial mass beyond a critical threshold to activate the nerve endings to precipitate an attack of BPPV. There are studies that have discussed the clinical entity "BPPV without nystagmus"-a condition in which attacks of vertigo occur with changes in the position of head in absence of an elicitable PN. ${ }^{3-8}$ Presumably on the basis of these studies reporting improvement in positional vertigo after a therapeutic positional maneuver in patients with a typical history of BPPV, the consensus document of the committee for the classification of vestibular disorders of the Bárány society has classified the cases of canalolithiasis of the posterior SCC without PN under the heading "Possible benign paroxysmal positional vertigo." It is believed that in such cases mass of the otoconial debris in the posterior SCC has not reached the critical threshold to stimulate the vestibulo-ocular reflex but is sufficient to evoke the vertigo with changes in the position of head relative to the gravity. ${ }^{9}$ In the Bárány society classification proposed in 2015, all other variants of BPPV without an elicitable PN, which are not attributable to posterior canalolithiasis, are lumped under an ambiguous heading "Probable benign paroxysmal positional vertigo, spontaneously resolved." However, the situations where a clinician examines a patient currently experiencing the typical symptoms of BPPV and fails to elicit a positioning nystagmus on positional tests (DHT and SRT) are extremely annoying. It is therefore imperative to try by all possible methods, to elicit a PN to precisely localize, as well as lateralize the diseased SCC before allowing an adjective like possible or probable being prefixed to the diagnosis of a disease whose diagnosis is entirely clinical. In a study of 81 patients diagnosed with unilateral PSC-BPPV, 12 patients who did not elicited PN initially on a conventional DHT were subjected to head shaking DHT (HSDHT), consisting of head shaking for five times with 30 degrees of excursion in the yaw plane with head maintained in the Dix-Hallpike position. All 12 patients who were subjected to HSDHT elicited upbeating torsional PN, increasing the diagnostic yield by $14.8 \% .^{10}$ The use of head-shaking in the yaw plane to unveil a horizontal positioning nystagmus in cases where a conventional positional test (DHT and SRT) has failed to elicit the PN has not been reported in the literature hitherto. The case number one described here, who historically appeared to have a left PSC-BPPV (history of vertigo on left and not on right lateral recumbent position and on assuming supine to sitting positions and vice versa), had initially failed to show PN on DHT and SRT. Thereafter, he was subjected to head-shaking in yaw plane in the left Dix-Hallpike position and the ensuing DHT and SRT triggered an apogeotropic and geotropic PN, respectively, finally leading to the diagnosis of left geotropic variant of HSC-BPPV. He was successfully treated with two sequences of Gufoni maneuvers at an interval of 1 hour, with follow-up at 1 hour and telephonically 24 hours after second sequence of Gufoni maneuver. The case number two described here, who historically appeared to have HSC-BPPV (vertigo episodes triggered by taking sitting to supine positions and vice versa as well as on taking either of the lateral recumbent positions), initially failed to show PN on SRT. In sitting position, he was subjected to head-shaking in yaw plane with head 30 degrees anteflexed in the pitch plane and the ensuing SRT after few attempts triggered bilateral geotropic horizontal PN (stronger on the right side), finally leading to the diagnosis of right geotropic variant of HSC-BPPV. He was successfully treated using Gufoni maneuvers, with follow-ups at 1 and 24 hours.

\section{Conclusion}

The diagnosis of BPPV is entirely dependent on the demonstration of PN on the diagnostic positional tests, namely DHT and SRT. It is extremely annoying for a clinician examining a patient currently experiencing paroxysms of vertigo triggered by the change in position of head but in whom meticulously performed positional tests do not 
elicit PN. In such a situation, it is imperative to re-examine the patient after some time. Head shaking during DHT has shown to increase the diagnostic yield of the PSC-BPPV in one observational study of 81 patients but there are no such previous reports or studies about HSC-BPPV. The cases presented here with supporting videos indicate that head shaking may unveil a PN not seen on conventional positional tests in cases of HSC-BPPV but more studies are required to prove this.

\section{Funding}

None.

\section{Conflict of Interest Statement}

None declared.

\section{Acknowledgments}

Thank you to Renith Kurian, videographer, for volunteering his time to make video recording of the diagnostic and therapeutic maneuvers and precisely capturing the nystagmus during the entire diagnostic and treatment period.

\section{References}

1 Dix MR, Hallpike CS. The pathology, symptomatology and diagnosis of certain common disorders of the vestibular system. Ann Otol Rhinol Laryngol 1952;61(4):987-1016

2 von Brevern M, Bertholon P, Brandt $\mathrm{T}$, et al. Benign paroxysmal positional vertigo: diagnostic criteria. J Vestib Res 2015;25(3-4):105-117
3 Tirelli G, D’Orlando E, Giacomarra V, Russolo M. Benign positional vertigo without detectable nystagmus. Laryngoscope 2001;111(6):1053-1056

4 Haynes DS, Resser JR, Labadie RF, et al. Treatment of benign positional vertigo using the semont maneuver: efficacy in patients presenting without nystagmus. Laryngoscope 2002;112(5):796-801

5 Anagnostou E, Mandellos D, Patelarou A, Anastasopoulos D. [Benign paroxysmal positional vertigo with and without manifest positional nystagmus: an 18-month follow-up study of 70 patients] HNO 2007;55(3):190-194

6 Zhang JH, Huang J, Zhao ZX, et al. [Clinical features and therapy of subjective benign paroxysmal positional vertigo]. Zhonghua Er Bi Yan Hou Tou Jing Wai Ke Za Zhi 2007;42(3):177-180

7 Johkura K, Momoo T, Kuroiwa Y. Positional nystagmus in patients with chronic dizziness. J Neurol Neurosurg Psychiatry 2008;79(12):1324-1326

8 Caldas MA, Ganança CF, Ganança FF, Ganança MM, Caovilla $\mathrm{HH}$. Clinical features of benign paroxysmal positional vertigo. Rev Bras Otorrinolaringol (Engl Ed) 2009;75(4):502-506

9 House MG, Honrubia V. Theoretical models for the mechanisms of benign paroxysmal positional vertigo. Audiol Neurotol 2003;8(2):91-99

10 Kaplan DM, Slovik Y, Joshua BZ, Puterman M, Kraus M. Head shaking during Dix-Hallpike exam increases the diagnostic yield of posterior semicircular canal BPPV. Otol Neurotol 2013;34(8):1444-1447 